\title{
Task-Layer Multiplicity as a Measure of Community Level Health
}

\author{
P. Fraundorf $\mathbb{i D}^{1,2}$ \\ ${ }^{1}$ Physics \& Astronomy/Center for Nanoscience Bldg., UMSL, St. Louis, MO 63121, USA \\ ${ }^{2}$ Physics Department, Washington University, St. Louis, MO 63110, USA \\ Correspondence should be addressed to P. Fraundorf; pfraundorf@umsl.edu
}

Received 23 January 2019; Accepted 4 June 2019; Published 1 July 2019

Academic Editor: Carlos Gershenson

Copyright (@ 2019 P. Fraundorf. This is an open access article distributed under the Creative Commons Attribution License, which permits unrestricted use, distribution, and reproduction in any medium, provided the original work is properly cited.

\begin{abstract}
The insights of many disciplines, and of commonsense, about individual-level well-being might be strengthened by a shift in focus to community-level well-being in a way that respects belief systems as well as the power of each individual. We start with the jargon of complex systems and the possibility that a small number of broken symmetries, marked by the edges of a hierarchical series of physical subsystem types, underlie the delicate correlation-based complexity of life on our planet's surface. We show that an information-theory-inspired model of attention-focus on correlation layers, which looks in/out from the boundaries of skin, family, and culture, predicts that behaviorally diverse communities may tend toward a characteristic task-layer multiplicity per individual of only $\mathrm{e}^{29 / 20} \cong 4.26$ of the six correlation layers that comprise that community. This behavioral measure of opportunity may help us to (i) go beyond GDP in quantifying the impact of policy changes and disasters, (ii) manage electronic idea-streams in ways that strengthen community networks, and (iii) leverage our paleolithic shortcomings toward the enhancement of community-level tasklayer diversity. Empirical methods for acquiring task-layer multiplicity data are in their infancy, although for human communities a great deal of potential lies in the analysis of web searches and asynchronous experience sampling similar to that used by "flu near you."
\end{abstract}

\section{Introduction}

Here we examine an empirical way to characterize the extent to which organisms generally, and people in particular, manage to spend time addressing matters that look inward, as well as outward, from their boundaries of skin, family, and culture. The target features of this approach are (i) a multilayer instead of a single-layer (e.g., economic-only) scope, (ii) a community-level rather than an individual (i.e., organismcentric) focus, and (iii) grounding in a cross-disciplinary view of emergent complexity.

The importance of a multilayer perspective was highlighted, for instance, by Francis Bacon [1] when reflecting on the correspondence between brotherhood in families, arts mechanical communalties, and religion and his proposed fraternity in learning and illumination (which in our parlance look, resp., in/out from the boundaries of family and culture). McShea recently pointed out [2] that nestedness is one of very few things that show quantitatively documented trends on the scale of life's history, and classic works, for example, by Okasha [3] as well as Richardson and Boyd [4], highlight the theme. A multilayer approach is illustrated more recently in the Gallup-Sharecare 5 Well-Being Index [5] and this note on such metrics in Science [6].

The need for a systems level approach (cf. [7]) is illustrated by the fact that discussions of both our intelligence and our well-being often center around individual organisms instead of community processes (cf. [8]) and that both community and individual measures of well-being face "a prodigious variety of preanalytic conditions" consistent with commonsense, along with an awareness of scientific insights across disciplines (cf. [9]). David Sloan Wilson puts it in Darwin's Cathedral [10]: “There was a time when individualism reigned supreme in both evolutionary biology and in the human social sciences, creating an image of the individual as the only adaptive unit (or rational actor) in nature and of the group as merely a byproduct of what individuals do to each other. Those days are over." 
Finally, cross-disciplinary views of emergent complexity often involve the following: (a) a relational versus a standalone subsystem focus (cf. [11]), that is, a concentration on subsystem interactions, (b) an order hierarchy predicated on gradients, boundaries, or edges that mark broken symmetries (cf. [12]), for example, between inside and outside a planet's atmosphere or a biological cell membrane, and (c) an inventory or pair/higher-order subsystem correlations (e.g., [13]) which look out/in, respectively, from such layered boundaries.

With or without robust theoretical underpinnings, of course, the selection of order parameters (e.g., [14]) for the upper layers of a complex-system hierarchy is likely to be a matter of field insight, plus trial and error. This is where crossdisciplinary field experience, lacking in this paper, will be crucial in the days ahead. Here we propose simply to examine the fractional attention that organisms can give to buffering correlations (i.e., relationships between subsystems) that look inward and outward from the three highest boundaries in the organizational hierarchy, namely, skin, family, and culture.

Correlation buffering here refers to an organism's natural role in preserving relationships and avoiding mismatches. For example, (i) life in a "food desert" may give rise to a mismatch between one's environment and needs felt inside one's skin, (ii) life in solitary confinement may give rise to a mismatch between one's environment and one's capacity for constructive pair interactions, (iii) geographic separation between parents and offspring may give rise to a mismatch between one's environment and one's ability to nurture family, (iv) displacement due to natural disaster (or economic opportunity) may result in the loss of community relationships and even loss of the skills that have been developed to maintain such relationships, (v) a cultural tradition's inability to adapt in today's changing information environment can result in loss of support and/or participants, and (vi) failure to respect other cultures or disciplines "in either direction" may result in loss of memetic diversity just as near-extinction of a species can result in loss of genetic diversity.

As we will see, the approach provides a framework for characterization and surprisingly robust goal formulation (e.g., to help balance a wide variety of differing individual perspectives). However, we will only know what is working if we have ways to obtain data on these matters. That will be the next challenge.

\section{An Optional Big Picture Context}

In the "natural history of invention," complexity emerges when specific information on broken symmetries, generally associated with gradients, boundaries, or pool edges, becomes available in the outside world. If and when an asymmetry (or external correlation with it, including external awareness of it) fades, the associated complexity fades along with it. Thus, for instance, liquid water might be seen as isotropic for all practical purposes, even though we know that on the nanoscale it has neither translational nor orientational symmetry.

One of the simplest examples of this is the Szilard vacuum-pump binary memory [15], in which a symmetric two-piston assembly with removable partition contains a single atom at an ambient-stabilized temperature $\mathrm{T}$, whose position can be "set" by removing the divider, inserting one piston using available work $W=k T \ln [2]$, followed by return of the partition and removal of the piston. We now know (i.e., have one bit of information about) which side the atom is on. We have added complexity to the world at cost of some thermodynamic availability.

That information can be irreversibly lost if we (i) remove and reinsert the partition, (ii) close our eyes and spin the assembly randomly about an axis through the partition, or (iii) forget which side we put the atom on. Thus at no cost, the world can become less complex. This exercise illustrates the "one-way" nature of spontaneous correlation loss, that is, of entropy increase and, the quantitative cost of complexity, that is, of correlation information between subsystems, plus several ways that complexity can spontaneously fade in the absence of effort to keep it in place. Thus, for example, faithfulness in replication of nucleic acid codes is a measure of their relevance to reproductive survival [16].

Earth life is part of the hierarchy of broken symmetries that began with the collapse of the solar nebula, the accretion of planetesimals to form the planet, and the formation of a surface boundary layer on that planet subjected to the flow of ordered energy (from within and without) to power a layered system of biogeochemical cycles. In these flows, shared-electrons first broke the symmetry between inmolecule and extra molecule interactions. In this context, many broken symmetries emerged and then faded, but the key symmetry breaks that we focus on here established a hierarchy of correlated subsystems made up of correlated subsystems.

Thus, one might be tempted to say that life began with the natural invention of bilayer membranes, whose closure allowed the break in symmetry between molecules inside and outside that membrane or cell wall. These singlecelled lifeforms can not only tolerate a much wider range of conditions than us multicelled organisms but they also invented digital storage of information in molecular codes.

Beyond that, shared resources (like steady-state flows) may have broken the symmetry between in-tissue and external processes, giving rise to our first multicelled organisms. Metazoan skins, in turn, allowed symmetry between inorganism and out-organism processes to be broken, bias toward family members broke the symmetry between infamily and extra familial processes, and membership-rules (e.g., tribal xenophobia) broke the symmetry between inculture and multicultural processes. The way that this layered hierarchy of subsystem correlations is supported by orderedenergy (or available-work) flows is illustrated in the left and center panels of Figure 1.

In this paper, we focus on the correlations with respect to the last three boundaries of most direct interest to metazoan individuals but not just on individual health or even on the health of whole family gene-pools (although this is a recent focus in biology, cf. [17]). In other words, we center our attention on the last three symmetry-break levels (skin, family, and culture) and the six subsystem-correlation layers associated therewith. 


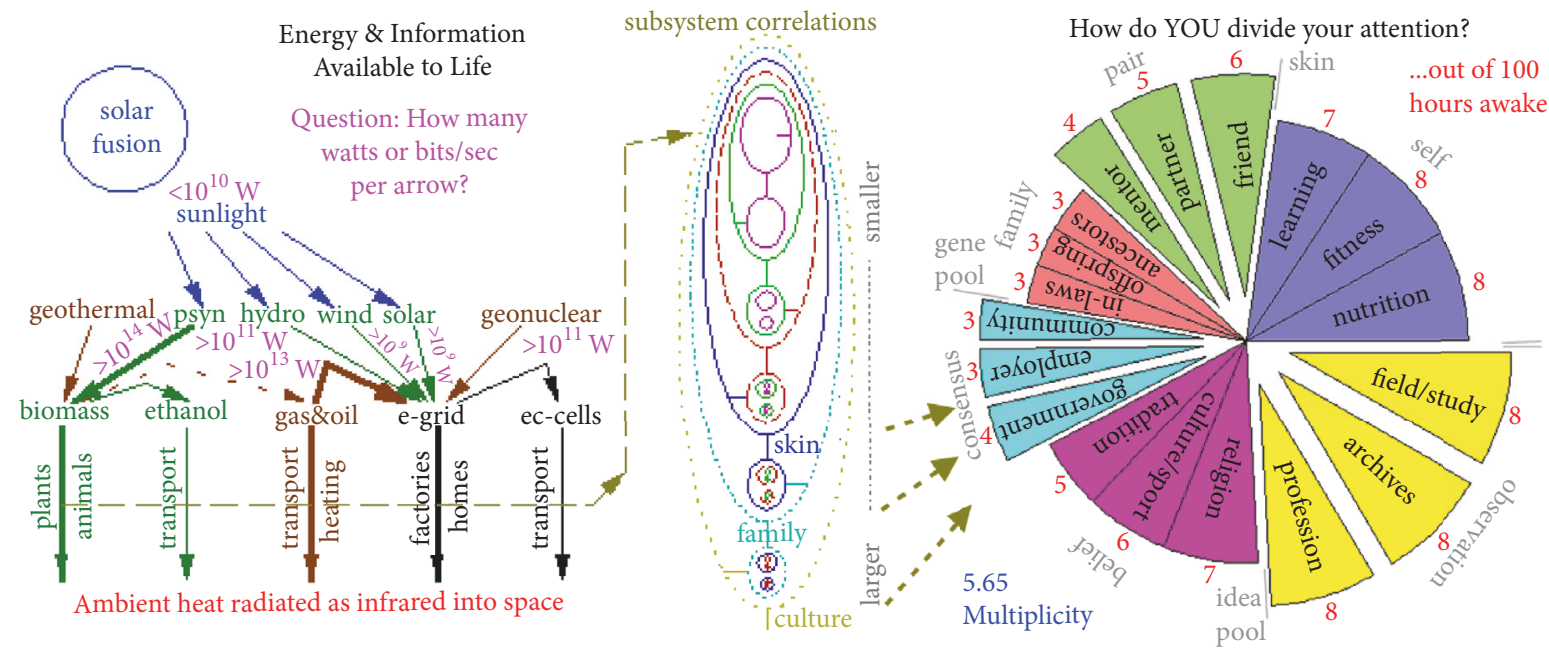

FIGURE 1: Available work flows (left) power a correlation hierarchy (center) that supports life's everyday complexity. At right, in this context, we illustrate one way to report results of an attention-slice survey for human individual, as well as community, task-layer multiplicity.
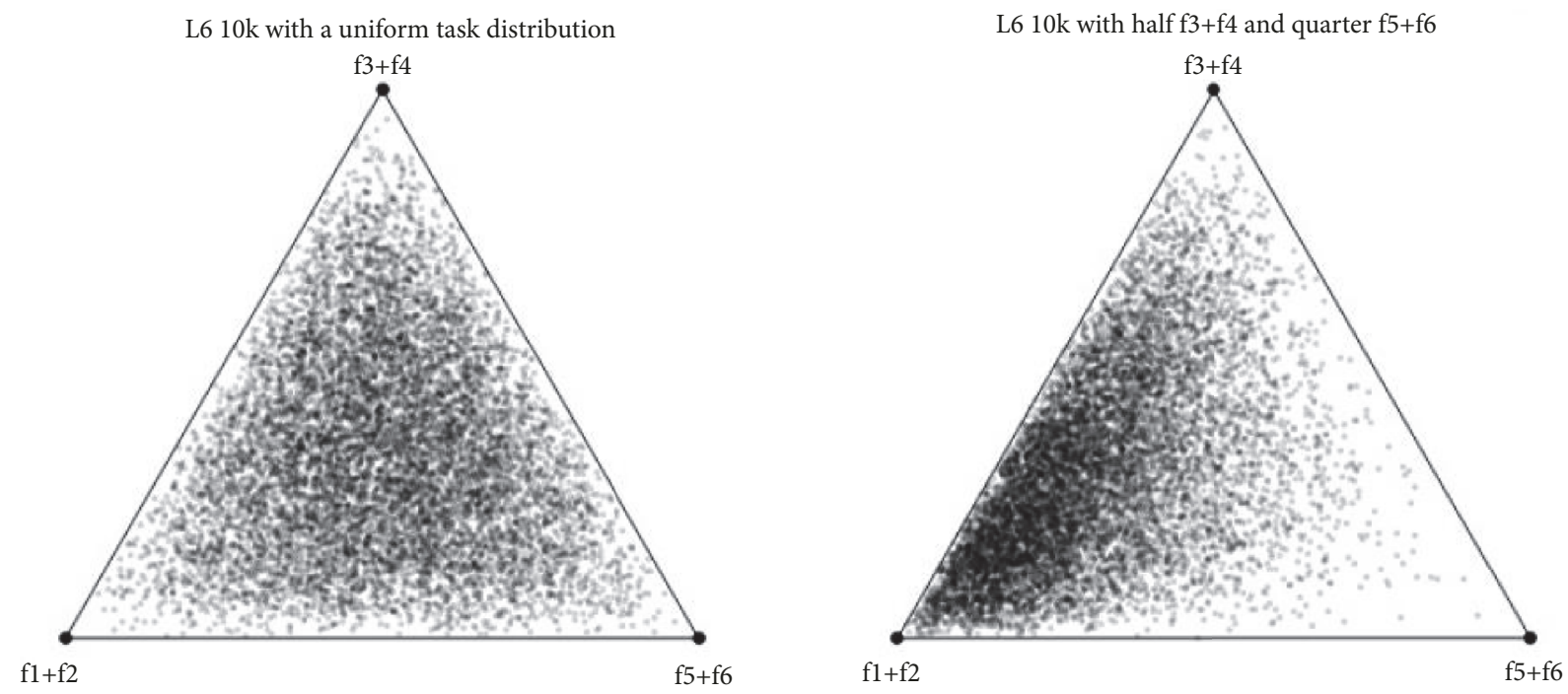

FIGURE 2: At left is a random simplex-point picked 6-layer population of 10,000 individuals, projected onto a ternary plot with subsystem correlations, for example, in/out from skin in the lower left, in/out from family at top, and in/out from culture at lower right, resulting in $M_{\mathrm{cm}} \sim$ 6 and $M_{\text {geom }} \sim 4.26$. At right is a similar 6-layer population, in which participation buffering of correlations that look in/out from family has been cut in half and of correlations that look in/out from culture has been divided by 4 , resulting in $M_{\mathrm{cm}} \sim 5.39$ and $M_{\text {geom }} \sim 3.87$. The latter might be expected, for example, for a human population that has limited access to jobs and even more-limited access to cultural/professional education.

\section{A Task-Layer Multiplicity Simplex}

Selection of order parameters for complex systems is sometimes more of an art than a science. Here, as in the selection of order-parameters for simpler (albeit still-complex) thermodynamic systems, we seek a measure based on information available with minimal disruption.

For inputs, we begin with (up to) $L=6$ normalized positive numbers $f_{\mathrm{i}}$ representing the fraction of an organism's effort allocated to buffering subsystem correlations associated with each of the 6 subsystem correlation-layers, that is, which look in/out from skin, family, and culture. In other words, by various means, we try to get a sense of the types of tasks that individuals in a given community manage to spend their time on. For visualization purposes, these six positive $f_{\mathrm{i}}$ values (which add up to 1) allow us to map the layer-focus of organisms to individual points within the unit 5-simplex between 6 vertices, just as ternary diagrams map any three normalized positive numbers onto an equilateral triangle or 2 -simplex in a plane. The latter in this context may be used to project normalized groups of these fractions, as shown in Figure 2, while a hexplot of ternary diagrams might be useful for a more complete view of an $L=6$ population (cf. Figure 3 ).

For inventory order, we then define a single metazoanindividual's niche-network layer-multiplicity $m$ as the behavior-defined effective number of correlation buffering 


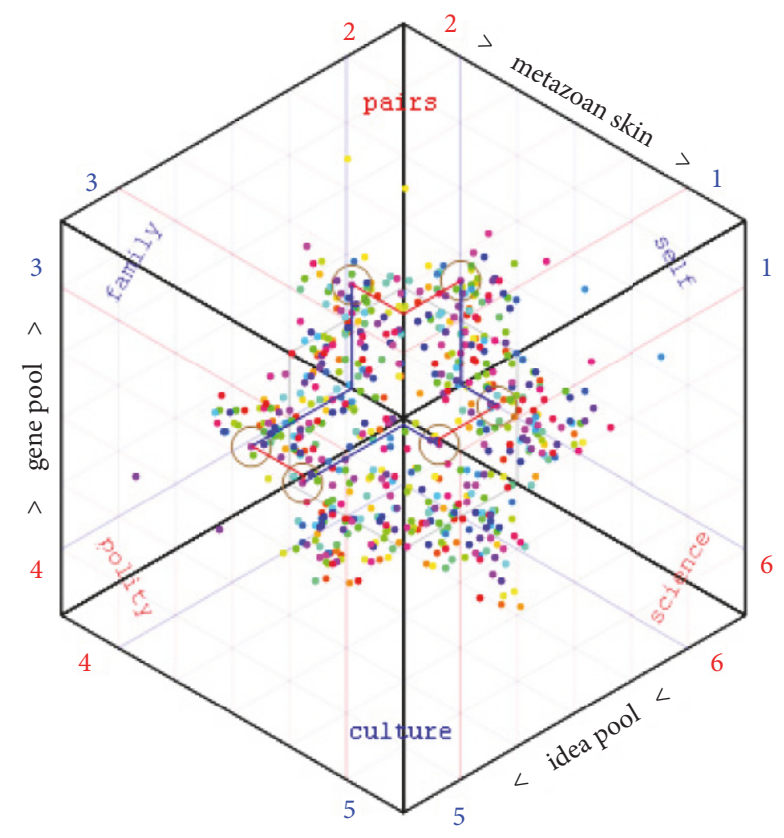

FIGURE 3: Six projections of 100-member random simplex pointpicked dot-cloud, with projections of one individual organism circled. The attention-fraction associated with the outer vertices is labeled, while the central vertex in each ternary-plot triangle represents the sum of the remaining fractions.

choices, expressed as an entropy-exponential in terms of that organism's set of, for example, $L=6$ fractional-attention values $\{f\}$ :

$$
1 \leq \#_{\text {choices }}=m[\{f\}]=\prod_{i=1}^{L}\left(\frac{1}{f_{i}}\right)^{f_{i}}=2^{\#_{\text {bits }}} \leq L
$$

where $\Sigma_{\mathrm{i}} f_{\mathrm{i}}=1$, that is, sums to one over the level index $i=1, L$.

This multiplicity measure can also be expressed in terms of the number of bits of surprisal [18] or state-uncertainty $S$ in bits about which correlation layer (e.g., self, friends, family, job, culture, and profession) they are working on at any given time; that is, $S=\ln _{2}[m]=\Sigma_{\mathrm{i}} f_{\mathrm{i}} \ln _{2}\left[1 / f_{\mathrm{i}}\right]$. However, use of $\#_{\text {choices }}$ instead of $\#_{\text {bits }}$ probably makes more sense here since the numbers are so small.

Population averages, that is, normalized sums over all $N$ community members (say using index $j=1, N$ ), will be denoted with angle brackets like \langle\rangle . Thus the average individual multiplicity is $\langle m\rangle=(1 / N) \Sigma_{\mathfrak{j}} m_{\mathfrak{j}}$, where $m_{\mathfrak{j}}$ is the task-layer multiplicity $m$ (as defined above) for the $j^{\text {th }}$ individual. The population average value for attention fraction $f_{\mathrm{i}}$ is $\left\langle f_{\mathrm{i}}\right\rangle=$ $(1 / N) \Sigma_{\mathrm{i}} f_{\mathrm{ij}}$, where $f_{\mathrm{ij}}$ is the $j^{\text {th }}$ individual's layer $i$ attention fraction.

We will use $\{\langle f\rangle\}$ to refer to the set of all $L$ attention fraction population averages. This allows us to define a centerof-mass multiplicity $M_{\mathrm{cm}}=\prod_{\mathrm{i}=1,6}\left(1 /\left\langle f_{\mathrm{i}}\right\rangle\right)^{\left\langle f_{\mathrm{i}}\right\rangle}$, representing the spread in attention focus for the community as a whole. In nonsocial organism communities, for instance, the fraction of time spent on matters of social hierarchy, let alone intraand extracultural pursuits, may be quite small, pushing the

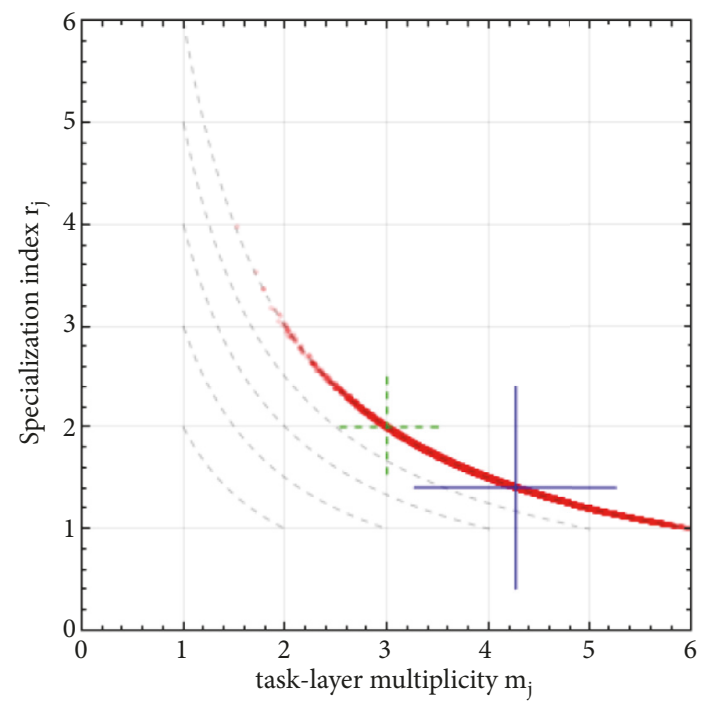

FIGURE 4: The red dots denote individual specialization indices $r_{\mathrm{j}}$ as a function of individual task-layer multiplicities $m_{\mathrm{j}}$ for organisms in a 6-layer random simplex point-picked population of 10,000 individuals. The blue cross is the specialization index $R$ for this population; the green-dashed cross is for a more specialized "nurture/adventure" population. The dashed lines follow $r_{\mathrm{j}} \sim L / m_{\mathrm{j}}$ for $L$ of 2 through 6 layers, successively outward from the origin.

center of mass multiplicity closer to only 3 of the 6 layers that we are considering here.

We may also want to consider average surprisal or entropy $\langle\mathrm{S}\rangle=(1 / N) \Sigma_{\mathrm{j}} \mathrm{S}_{\mathrm{j}}$ for $\mathrm{j}$ running from 1 to $N$. This leads simply to the geometric average individual multiplicity, defined as $M_{\text {geom }}=2^{\langle\mathrm{S}\rangle}=\left(\Pi_{\mathrm{j}} m_{\mathrm{j}}\right)^{1 / N}$, for which it is easy to show that $M_{\text {geom }} \leq M_{\mathrm{cm}}$. Because of this organic relation to the centerof-mass value, we will use $M_{\text {geom }}$ as our indicator of the spread in attention focus for individual organisms within the community. For instance, a community of individuals might have a center of mass multiplicity of 6 even if half of the individuals only take on nurturing (e.g., inward-looking or post-pair correlation) tasks, while the other half only take on adventuring (i.e., outward-looking) tasks. In that case, the geometric average multiplicity would only be about 3 .

The inequality above naturally lets us define organism and community specialization indices, whose logarithms are KLdivergences or relative entropies, that is, the always-positive entropy generalization (cf. [19]) that here employ the set of $\{\langle f\rangle\}$ values as the reference correlate. These indices decrease in value toward 1 only as the spread of individual foci begins to match that of the community as a whole. For the community specialization index $R$, we use $1 \leq R \equiv M_{\mathrm{cm}} / M_{\text {geom }} \leq M_{\mathrm{cm}}$. The community specialization index $R$ would thus be only about 1 for a community in which all individuals spent equal amounts of time on all six layers, while for a community adopting the "nurture/adventure" (or "yin/yang") dichotomy mentioned above, the specialization index would approach 2 .

For use only in Figure 4, although they are also useful for deriving some inequalities, along with individual multiplicity $m_{\mathfrak{j}}$ defined above one might also define individual 
specialization indices $r_{\mathrm{j}}=\Pi_{\mathrm{i}}\left(\mathrm{f}_{\mathrm{ij}} /\left\langle\mathrm{f}_{\mathrm{i}}\right\rangle\right)^{f_{\mathrm{ij}}}$. Like the community specialization index $R, r_{\mathrm{j}}$ will always be between 1 and $L$.

Finally, we recommend comparison of communities in this context with a "uniform-reference" community, in which all combinations of task assignment are equally probable. In general, this will allow researchers to see operating biases toward effort spent buffering subsystem correlations on one layer or another. Comparison of experimental data from real communities, to this reference, might also help explore the possibility that task-layer diversity has a selective advantage and/or is a useful measure of community well-being. Quantitative aspects of this reference are discussed further in the Appendix.

\section{Applications}

Describing live communities quantitatively in terms of subsystem correlations may be in its infancy. Operational models for describing subsystem correlations in biofilms, within and between species in plant communities, in communities of social insects, as well as in primate communities including our own, can only be done with help from experts with field involvement in each of these areas.

The objective of this section is therefore simply to take a cursory look at some aspects of the potential for such an approach, with a bias toward its application in 6-layer human communities. Moreover, we will focus mainly on uses not for detailed aspects of observed distributions but on center-ofmass task-layer multiplicity $M_{\mathrm{cm}}$ as a measure of correlationlayer activity relevant to the survival of living systems and the perhaps more subtle adaptive value of task-layer diversity, that is, of a community with specialists and generalists of all sorts. These analyses treat all subsystem-correlation layers equally, in spite of a hierarchical structure that shows they are not (in other words, individuals are clearly prerequisite to family, which in turn may be prerequisite to culture). By averaging over any given community's population, data in this form is perhaps also by its nature "anonymous" as far as specific individuals in a community are concerned, even though establishing useful protocols for obtaining it in any given community type remains a future challenge to be discussed briefly in the next section.

4.1. Task-Layer Breadth. Imagine that $M_{\mathrm{cm}}$ began increasing toward 2 when the metazoan skin of multicelled organisms predicated the symmetry-break between self-focused behaviours (like hunger and fear) and pair-focused behaviours (like aggression and pair-bonding). When such social organisms began treating their young differently from the young of others, molecular code-pool boundaries facilitated the symmetry-break between family-focused behaviours (like bower-building and child-rearing) and socially focused behaviours (like status-pursuit and community-service) letting $M_{\mathrm{cm}}$ approach 4 . Center-of-mass multiplicity $M_{\mathrm{cm}}$ was allowed to approach 6 only after communicating organisms began recognizing distinctions between in-group and outsider patterns, allowing idea-pool symmetry-breaks to distinguish behaviours that are culturally focused (like religion and sports) and extracultural (like professional-development and library-building). Astrophysical observations indicate that environments for such multilayer correlation structures are short-lived (e.g., [20]), so quantitative models for $M_{\mathrm{cm}}$ 's increase and decrease with time may be worthwhile.

These models might provide integrative measures of social patterns already of interest like division of responsibility between large and small gamete metazoans (i.e., female/male role specialization) and quantitative comparison of the extent and nature of community cultural correlations from one species to another or from one time to another for a given species. If center-of-mass multiplicity correlates with other measures of health in human communities, it could be especially important for going beyond single-layer measures, like gross domestic product and body count, for taking quantitative account of family and culture when assessing the impact of policy changes and disasters on a given community (cf. Figure 2).

There are immediate as well as abiding practical possibilities here. Available resources, as well as the preservation of task layer-diversity, mean that individual humans are fallible in that their capabilities will either span only a part of the 6-layer correlation-hierarchy that underlies human social-systems today or be spread quite thin across all 6 . This is also true, in spite of our evolutionary attraction to social hierarchies, about the vision of any given leader or demagogue.

Regardless, as the ordered energy available per capita decreases (with either increasing population or energy costs), we can expect the 6-layer structure of our social systems to experience pressure to deconstruct (e.g., [21]). The demagogues of communism and fascism in the last century, as well as the demagogues of religious fundamentalism today, are evidence of pressure to toss out one layer or another of our social organization. Data with which to track, and concepts with which to communicate, about these pressures and their effects may be important if we want to give human social systems on earth a chance to do their best.

4.2. Task-Layer Diversity. When diversity of task assignments for individuals, as distinct from the task-layer breadth of attention in the community as a whole, is maximized by random simplex point-picking as outlined in the Appendix, $M_{\mathrm{cm}} \sim 6$ but $M_{\text {geom }} \sim 4.26$. In other words, the opportunity to be equal may not argue that everyone contributes on all layers (specialization index $R \sim 1$ ). However, we might look for a specialization index closer to 1.4 , for example, significantly less than $R \sim 2$ expected for a community with "nurture/adventure" (sometimes cast, e.g., as "female-male") role-specialization. This may help us address the "urgent question" posed in the late 19th century by Emile Durkheim in his dissertation on workplace divisions of labor [22], whether to choose roundedness or specialization, by saying "if possible explore roundedness, but specialize when that works better for you." This is consistent with subsequent trends away from rigid divisions of labor (e.g., based on heritage and gender) at home as well as at work.

The physiological division of labor between large and small gamete metazoans in reproductive roles, for example, in social insect communities, shows that task-layer diversity 
may not always be an adaptive choice. However, communities with higher free energy per capita and electronic information flow seem to be moving away from cultural role-divisions. Figure 4 illustrates by comparing $R$ and $M_{\text {geom }}$ of a 6-layer model with task diversity maximized by random simplex point-picking (larger plus) with the same quantities for a "yin-yang" community (smaller plus) in which half of the organisms each buffer subsystem correlations directed only inward, or only outward, from skin, family, and culture.

\section{The Data Challenge}

All of the applications above are predicated on a source of data about resource-allocation or, perhaps more simply, metazoan attention focus in a given single-species community. Resource allocation toward correlations looking in/out from skin, family, and culture may be impossible to quantify objectively, but "time on task" may serve as a stand in, as illustrated, for example, for human communities in the rightmost panel of Figure 1.

One may attempt to acquire data on some organism communities by direct observation. In human communities, however, voluntary self-reporting and communication-traffic analysis may both be more accessible and more respectful of individual privacy, particularly for data on short-term changes in attention focus. Such an early effort at such selfreporting involved Hadley Cantril's work on "the pattern of human concerns" [23]. In fact, the measure discussed here might be seen as an attempt to add structure to those concerns, anchored in insights about the bloom and decline of complexity in the natural world.

Modern self-reporting strategies might involve search activity (data on patterns of curiosity freely given in return for information) or even experience-sampling [24, 25] like that of "flu near you" [26] or by asking participants to select a layer from 1 to 6 on your phone, when the occasional request comes in. In fact, the community well-being categories in the Gallup-Healthways Well-Being 5 Index [5] might be seen as mapping loosely to correlations that look inward from skin ("physical"), inward from family ("social"), outward from family ("financial"), outward from skin ("community"), and in/outward from culture (combined, e.g., as belief and profession related "purpose").

\section{Conclusions}

In this paper, we describe a "broken-symmetry" approach directed toward the description of structure in metazoan communities, grounded in common sense, as well as insights from the physical, natural, and social sciences. Given further work on ways to gather data, the measure might be useful for monitoring the bloom and decline of complexity in single species and especially human communities. It might also be used to monitor the impact of disasters and policy changes on "community" as distinct from individual health.

Perhaps we should close with a reflection on the tension between the "individual happiness" industry [27] and a focus instead on one's individual impact on community well-being. Individual glorification through social media is by and large a recipe for shallow commitment to others, to the celebrity of few among many, and hence to depression. In fact, in the move toward communities structured to support more than just service to your employer, contributions to community task-layer multiplicity (if measurable) might serve as the economic basis for sustainable communities with a broadly conceived but accountable reward system (and safety net) for individual participants.

Beyond this, as we turn our focus on a finite planet to sustainability, connections of individual well-being to our understanding of the gain and loss of complexity in both physical and biological systems will of course still be important. By way of example, Cloninger's measures [28] of unconscious style or temperament seem largely physiological, but his conscious "idea-mediated" elements of character (namely, self-regulation, cooperativeness, and judicial-transcendence as more active elements of our "postpaleolithic" development) might map reasonably well with our interest in one's attention focus on broken-symmetry subsystem correlations that look in/out, respectively, from skin, family, and culture. Clearly, experts from more than one field are called upon to acquire and explore data relevant to possible connections like this and more importantly to put such connections to good use. To this end, some space for links to related experience sampling sites, as well as to development code for analyzing task-layer multiplicity data after the fact, has been set up on the web [29].

\section{Appendix}

\section{The Uniform Task-Layer Diversity Reference}

A nice mathematical feature of simplex models, involving normalized fractions or probabilities, is that they follow the statistics of compositional analysis (cf. [30]). This means that the statistics is already well explored, and it makes projections from a 5-simplex with 6 vertices into lower-dimensional simplex spaces easy as well (cf. Figures 2 and 3). Hence a wide range of understandable illustrations, for example, of the effect of policy changes and events on a community's focus can be expected as more data on real communities in this format, become available.

For the moment, in order to explore an L-layer community in which all possible mixes of attention focus for individuals occur with equal probability, we examined analytical approaches, as well as algorithms for random simplex-point picking based, for example, on the Dirichlet distribution (cf. Figure 5). When running these algorithms on say 100 communities each of a million individuals, they all predict that the center-of-mass multiplicity approaches L, since there is no bias in this random model toward effort directed toward one layer of community organization over another. In other words, we expect the population average for attention fraction $f_{\mathrm{i}}$ to equal $1 / L$.

This reference value (denoted with an asterisk) for a 6layer community of $M_{\mathrm{cm}}{ }^{*} \sim 6$ thus signifies the collective ability of the community to apportion its effort equally toward the buffering of correlations that look in/out from skin, family, and culture. Limited historical opportunities, 


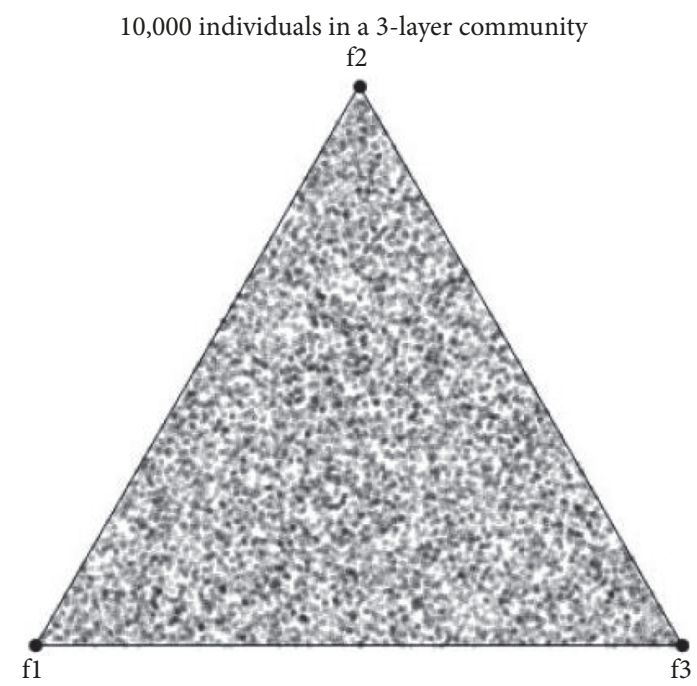

FIGURE 5: This is a test of our Dirichlet-based routine for random simplex-point picking, using a unit 2-simplex with 3 vertices, because the uniformity associated with 10,000 points is easily illustrated on a flat-screen ternary diagram.

policy changes, disasters, and environmental changes can only reduce this value.

The foregoing quantity, however, says nothing about rolespecialization or the lack thereof. For instance, one might think of social-insect communities with extreme amounts of role-specialization, but which nonetheless manage to buffer correlations on all the levels needed for their survival. One way to measure this is to look at the breadth of activities for individuals in the community. Rather than measuring diversity against a requirement that "all individuals give equal effort in all layers," however, we propose here that we look for biases in experimental data with respect to a community in which (as above) all possible task assignments are equally probable. This kind of reference should help examine biases for or against any type of task-layer assignment.

Following rigorous derivation of $M_{\text {geom }}{ }^{*}$ for communities with $L \leq 3$, we infer that a uniform distribution of tasks for arbitrary $L$ will give

$$
M_{\text {geom }}{ }^{*}=2^{\int_{0}^{1} d f_{1} \int_{0}^{1-f_{1}} d f_{2} \ldots \int_{0}^{1-\sum_{i=1}^{L-2} f_{i}} d f_{L-1}(L-1) ! S}
$$

where as usual $\mathrm{S}=\ln _{2}\left[\sum_{\mathrm{i}=1}^{L} f_{\mathrm{i}}^{f \mathrm{i}}\right]$ and $f_{L}=1-\sum_{\mathrm{i}=1}^{L-1} f_{\mathrm{i}}$. This implies that, for communities of one to eight layers,

$$
\begin{aligned}
& M_{\text {geom }} \\
& =\left\{1, e^{1 / 2}, e^{5 / 6}, e^{13 / 12}, e^{77 / 60}, e^{29 / 20}, e^{223 / 140}, e^{481 / 280}\right\}
\end{aligned}
$$

This assertion has been checked quantitatively to half dozen significant figures for values through $L=6$ by simplex-point picking and suggests that a good rule of thumb (for $L \leq$ 10 within $0.5 \%)$ is $M_{\text {geom }}{ }^{*} \sim 0.65 L+0.35$. Thus unbiased distribution of task assignments in an $L=6$ community means that individuals on average are buffering subsystem correlations in only $\mathrm{M}_{\text {geom }}{ }^{*}=\mathrm{e}^{29 / 20} \sim 4.2631$ layers. This is good news, given that the opportunity to buffer more layers was probably absent during the paleolithic times of our species' evolution. It is also good news for individuals in that, even when the opportunity to "do everything" is available, it may well not be your best choice.

\section{Data Availability}

Literature data used to support the findings of this study (which make a case for the importance of targeted data collection downstream) are cited with the article.

\section{Disclosure}

This paper is only corollary to (and hence not explicitly funded by) my research and regional outreach with students at the University of Missouri Saint Louis, in electron-beam characterization of materials, in Bayesian data analysis, and in the modernization of physics-education content.

\section{Conflicts of Interest}

The author declares that there are no conflicts of interest regarding the publication of this paper.

\section{Acknowledgments}

Thanks are due to the late Ed Jaynes and Myron Tribus for their enthusiasm about statistical inference and also due to Zak Jost for many careful readings during this project.

\section{References}

[1] F. Bacon, The Advancement of Learning, Cassell \& Company, New York, NY, USA, 1605.

[2] D. W. McShea, "Unnecessary complexity," Science, vol. 342, pp. 1319-1320, 2013.

[3] S. Okasha, Evolution and the Levels of Selection, Oxford University Press, 2008.

[4] P. J. Richerson and R. Boyd, Not by Genes Alone, U. Chicago Press, 2004.

[5] L. E. Sears, S. Agrawal, J. A. Sidney et al., "The well-being 5: development and validation of a diagnostic instrument to improve population well-being," Population Health Management, vol. 17, no. 6, pp. 357-365, 2014.

[6] C. Graham, K. Laffan, and S. Pinto, "Well-being in metrics and policy," Science, vol. 362, no. 6412, pp. 287-288, 2018.

[7] N. Luhmann, "The world society as a social system," International Journal of General Systems, vol. 8, no. 3, pp. 131-138, 2008.

[8] S. Sloman and P. Fernbach, The Knowledge Illusion: Why We Never Think Alone, Riverhead Books, New York, NY, USA, 2017.

[9] M. A. Bishop, The Good Life: Unifying the Philosophy and Psychology of Well-Being, Oxford University Press, 2015.

[10] D. S. Wilson, Darwinś Cathedral, University of Chicago Press, Chicago, IL, USA, 2002.

[11] C. Rovelli, Seven BriefLessons on Physics, Riverhead Books, New York, NY, USA, 2016.

[12] P. W. Andersen, “More is different," Science, vol. 177, no. 4047, pp. 393-396, 1972. 
[13] E. Schneidman, M. J. Berry II, R. Segev, and W. Bialek, "Weak pairwise correlations imply strongly correlated network states in a neural population," Nature, vol. 440, no. 7087, pp. 1007-1012, 2006.

[14] J. P. Sethna, Entropy, Order Parameters, and Complexity, Oxford University Press, 2006.

[15] L. Szilard, "Uber die Entropieverminderung in einem thermodynamischen system bei eingriffen intelligenter wesen," Zeitschrift für Physik, vol. 53, no. 11-12, pp. 840-856, 1929.

[16] G. D. Stormo, "Information content and free energy in DNA-protein interactions," Journal of Theoretical Biology, vol. 195, no. 1, pp. 135-137, 1998.

[17] M. A. Nowak, C. E. Tarnita, and E. O. Wilson, "The evolution of eusociality," Nature, vol. 466, no. 7310, pp. 1057-1062, 2010.

[18] M. Tribus, Thermostatics and Thermodynamics, D. Van Nostrand Co., Princeton, NJ, USA, 1961.

[19] P. C. Gregory, Bayesian Logical Data Analysis for the Physical Sciences: A Comparative Approach with Mathematica Support, Cambridge University Press, Cambridge, UK, 2005.

[20] P. D. Ward and D. Brownlee, The Life and Death of Planet Earth, Copernicus, New York, NY, USA, 2000.

[21] E. J. Chaisson, "Complexity: an energetics agenda," Complexity, vol. 9, no. 3, pp. 14-21, 2004.

[22] E. Durkheim, De La Division Du Travail Social, Presse Universitaires de France, 1893.

[23] H. Cantril, The Pattern of Human Concerns, Rutgers University Press, New Brunswick, NJ, USA, 1965.

[24] J. M. Hektner, J. A. Schmidt, and M. Csikszentmihalyi, Experience sampling, Thousand Oaks, 2007.

[25] M. A. Killingsworth and D. T. Gilbert, "A wandering mind is an unhappy mind," Science, vol. 330, no. 6006, p. 932, 2010.

[26] K. Baltrusaitis, M. Santillana, A. W. Crawley, R. Chunara, M. Smolinski, and J. S. Brownstein, "Determinants of participants' follow-up and characterization of representativeness in flu near you, a participatory disease surveillance system," JMIR Public Health and Surveillance, vol. 3, no. 2, p. e18, 2017.

[27] W. Davies, The Happiness Industry, London, UK, Verso Books, 2015.

[28] R. C. Cloninger, Feeling Good: The Science of Well-Being, Oxford University Press, 2004.

[29] “Task Layer Multiplicity \& Community Well Being," https:// sites.google.com/a/umsl.edu/tlm/.

[30] J. Aitchison, The Statistical Analysis Of Compositional Data, Chapman and Hall, London, UK, 1986/2003. 


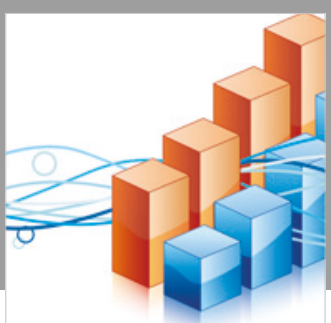

Advances in

Operations Research

\section{-n-m}
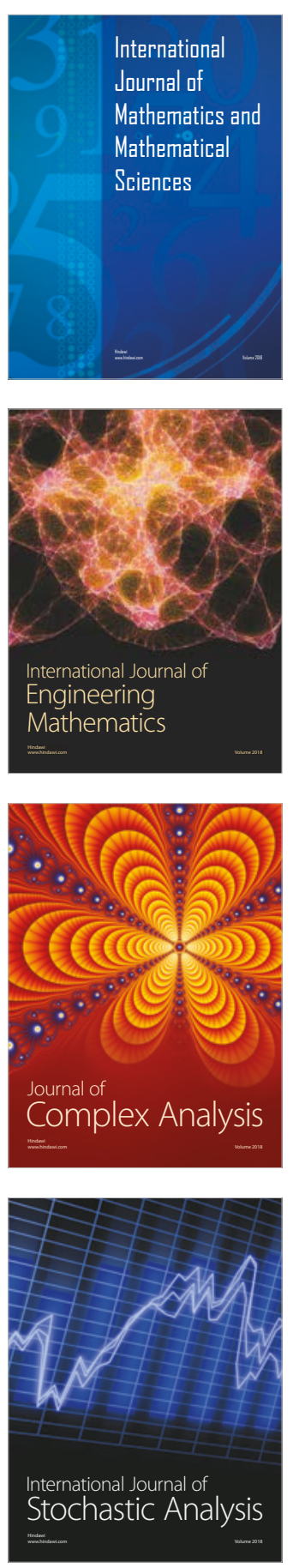
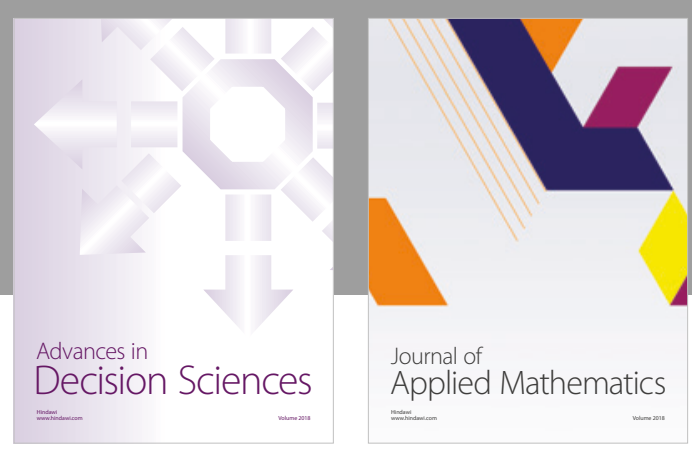

Journal of

Applied Mathematics
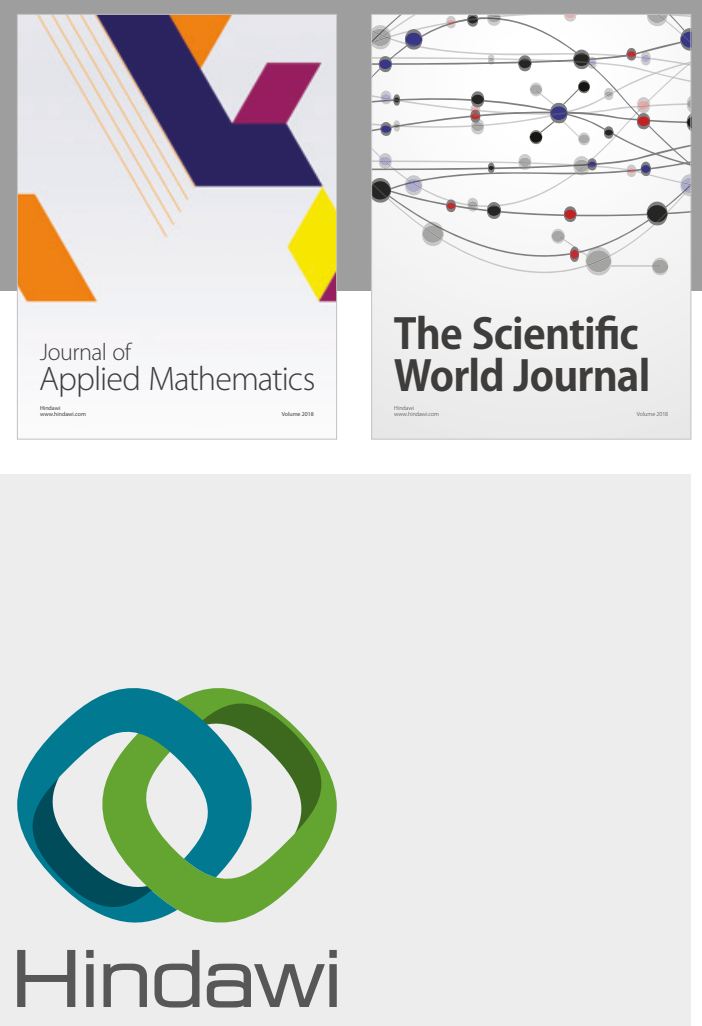

Submit your manuscripts at

www.hindawi.com

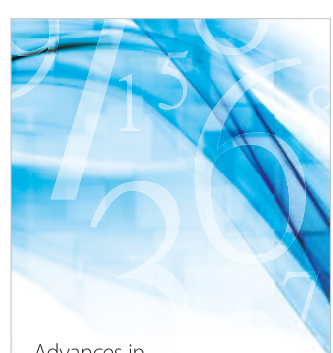

Advances in
Numerical Analysis
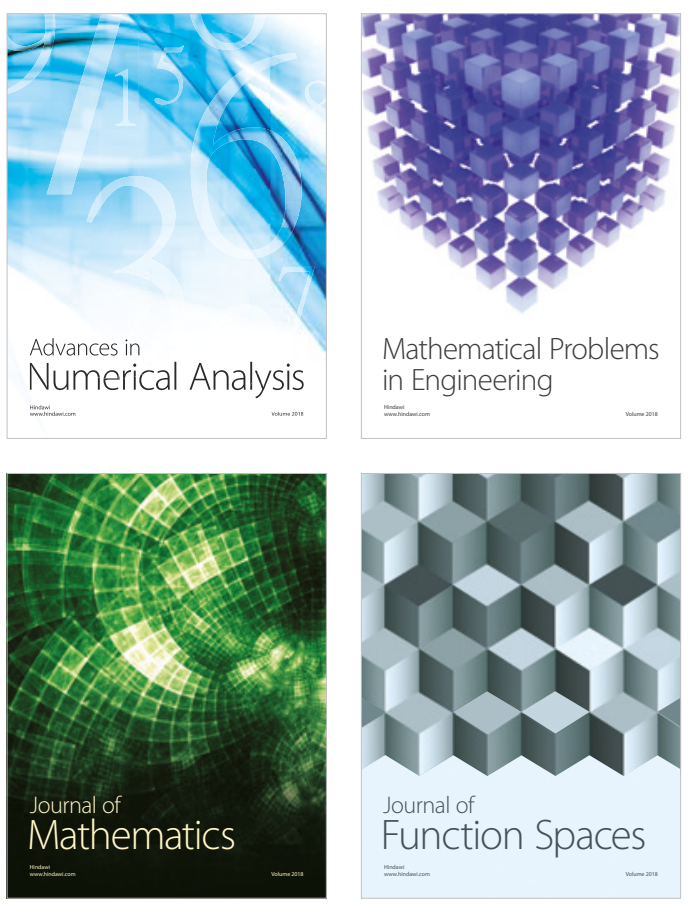

Mathematical Problems in Engineering

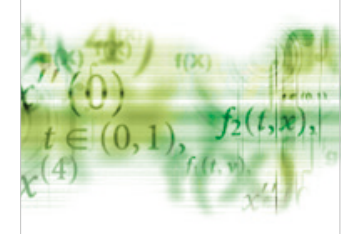

International Journal of

Differential Equations

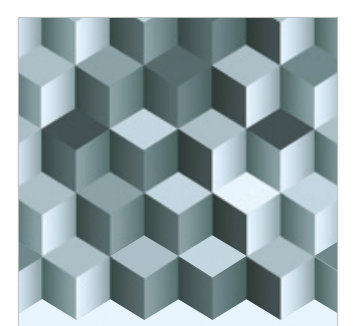

Journal of

Function Spaces
The Scientific

World Journal

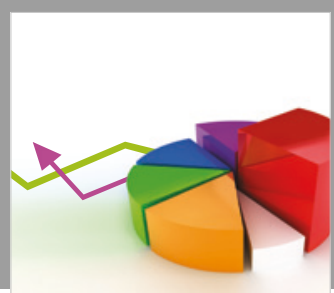

Journal of

Probability and Statistics
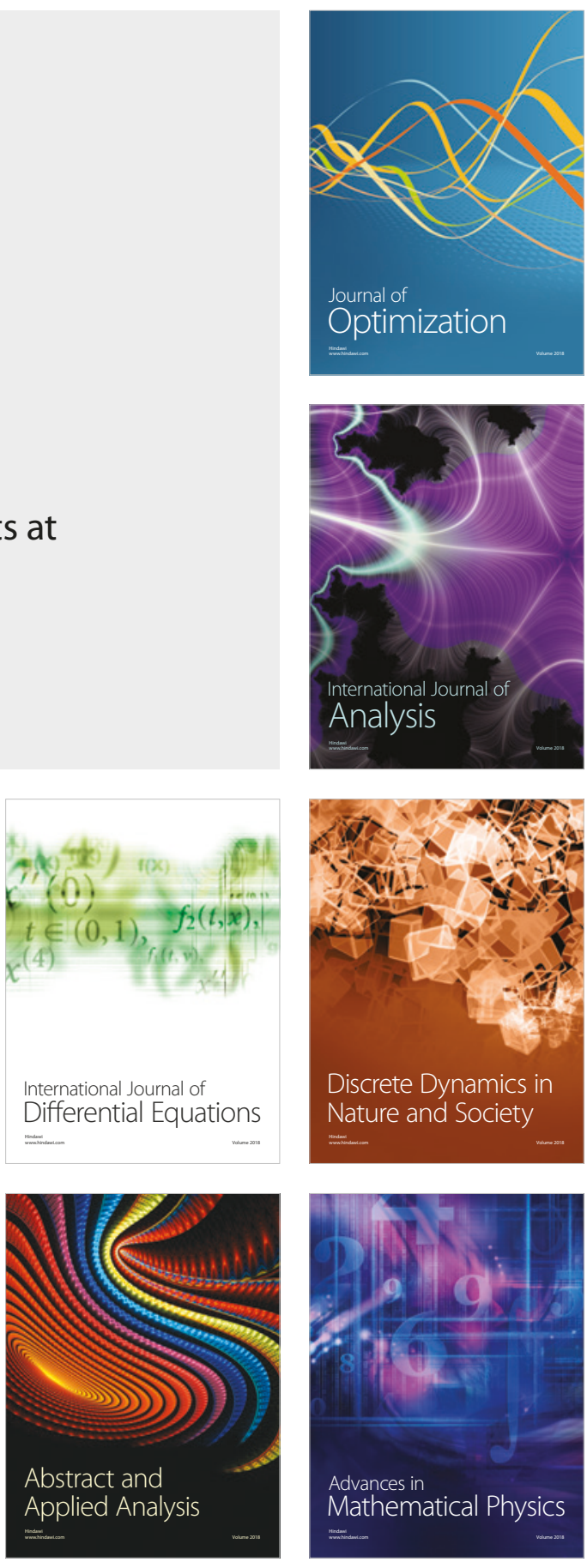